\title{
NQO1 protein expression predicts poor prognosis of non-small cell lung cancers
}

\author{
Zhenling Li ${ }^{1 \dagger}$, Yue Zhang ${ }^{2 \dagger}$, Tiefeng Jin ${ }^{1}$, Jiguang Men ${ }^{3}$, Zhenhua Lin ${ }^{1}$, Peng Qi ${ }^{3}$, Yingshi Piao ${ }^{1,4^{*}}$ and Guanghai Yan ${ }^{1,3^{*}}$
}

\begin{abstract}
Background: High-level expression of $\mathrm{NAD}(\mathrm{P}) \mathrm{H}$ : quinoneoxidoreductase 1 (NQO1) has been correlated with many types of human cancers, suggesting that NQO1 plays important roles in tumor occurrence and progression. This study attempted to explore the role of NQO1 in tumor progression and prognostic evaluation of non-small cell lung cancer (NSCLC).

Methods: Total 164 tissue samples, including 150 NSCLC paired with the adjacent non-tumor tissues and 14 normal lung tissues, were picked-up for immunohistochemical (IHC) staining of the NQO1 protein, and immunofluorescence (IF) staining was also performed to detect the subcellular localization of the NQO1 protein in A549 human lung cancer cells. The correlation between NQO1 expression and clinicopathological characteristics were evaluated by Chi-square test and Fisher's exact tests. The disease-free survival (DFS) and overall survival (OS) rates of NSCLC patients were calculated by the Kaplan-Meier method, and univariate and multivariate analyses were performed using the Cox proportional hazards regression model.

Results: The NQO1 protein showed a mainly cytoplasmic staining pattern in lung cancer cells, including adenocarcinoma and squamous cell carcinoma (SCC). Both positive rate and strongly positive rate of NQO1 protein expression were significantly higher in NSCLC (59.3\% and 28.0\%) than that in adjacent non tumor (8.0\% and 1.3\%) and normal lung tissues (0\%). The positive rate of NQO1 was related with clinical stage and lymph node metastasis, and the strongly positive rate of NQO1 protein was significantly correlated with tumor size, poor differentiation, advanced clinical stage and lymph node metastasis in NSCLC. Additionally, survival analyses showed that the patients with NQO1 positive expression had lower OS rates compared with those with NQO1 negative expression in the groups of T1-2, T3-4, without LN metastasis and stage I-II of NSCLC, respectively; however, in the groups of patients with LN metastasis or III-IV stages, OS rate was not correlated with NQO1 expression status. Moreover, multivariate analysis suggested that NQO1 emerged as a significant independent prognostic factor along with tumor size, differentiation, lymph node metastasis and clinical stage in patients with NSCLC.
\end{abstract}

Conclusions: $\mathrm{NQO} 1$ is upregulated in NSCLC, and it may be a useful poor prognostic biomarker and a potential therapeutic target for patients with NSCLC.

Keywords: Non-small cell lung cancer, NQO1, Immunohistochemistry, Prognosis, Survival analysis

\section{Background}

Non-small cell lung cancer (NSCLC) accounted for approximately $85 \%$ of all lung cancers, and it is the most common cause of death in both men and women [1]. Currently, molecular target therapy is one of the promising field of NSCLC treatment, and its target includes

\footnotetext{
* Correspondence: yspiao@ybu.edu.cn; guanghyan@hotmail.com

${ }^{+}$Equal contributors

'Department of Pathology \& Cancer Research Center, Yanbian University Medical College, Yanji 133002, China

Full list of author information is available at the end of the article
}

epidermal growth factor receptor (EGFR) and echinoderm microtubule associated protein like4-anaplastic lymphoma kinase (EML4-ALK). EGFR tyrosine kinase inhibitor (EGFR TKI, such as gefitinib and erlotinib) and EML4/ALK inhibitor (Crizotinib) have achieved better results in the clinical therapy of advanced NSCLC $[2,3]$. Despite progress in the multimodality treatment of lung cancer, prognosis is still poor, with 10-15\% 5-year survival rates. More than $90 \%$ of deaths from NSCLC are attributable to metastases $[1,4]$.

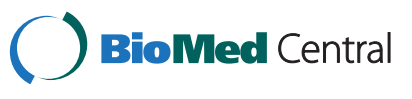


NAD(P)H: quinone oxidoreductase 1 (NQO1, EC 1.6.99.2) is well known as DT-diaphorase, and it can protect cells against radiation and chemical-induced oxidative stress. NQO1 is a cytosolic flavoenzyme that catalyzes the obligatory two-electron reduction of a variety of quinone substrates by using NADH or NADPH as electron donors [5]. And several functions of NQO1 have been found, such as xenobiotic detoxification, superoxide scavenging, modulation of p53, maintenance of endogenous antioxidants, and proteasomal degradation [6]. Due to the ability of NQO1, it is imaginable that NQO1 may play an important role in protecting normal cells against oxidative damage and electrophilic attack $[7,8]$. Recent studies reported that NQO1 is mainly expressed in cytosol, and low expression levels have been found in the nucleus. Moreover, NQO1 was found to be expressed at high levels in many human cancers, including liver, colon, pancreas and cholangiocarcinoma [9-12]. Garate et al. [13] indicated that the expression of NQO1 protein significantly induced cell cycle progression and led to the proliferation of melanoma cells by the up-regulation of cyclin A2, B1 and D1. However, the role of NQO1 in progression of lungcancer cells remains unidentified, and the correlation between NQO1 expression and NSCLC has not been adequately elucidated yet.

To determine whether NQO1 is important in the tumorigenesis of NSCLC and investigate the prognostic value of NQO1 expression level, total 150 cases of NSCLC paired with the adjacent non-tumor tissues and 14 of normal lung tissues were selected for NQO1 IHC staining. Our data uncover that NQO1 is frequently upregulated in NSCLC compared with the normal counterpart, and suggest that NQO1 may be an independent biomarker for prognostic evaluation of patients with NSCLC.

\section{Methods}

\section{Ethic statement}

This research complied with the Helsinki Declaration and was approved by the Human Ethics Committee and the Research Ethics Committee of Yanbian University Medical College. Patients were informed that the resected specimens were stored by the hospital and potentially used for scientific research, and that their privacy would be maintained. Follow-up survival data were collected retrospectively through medical record analyses.

\section{Clinical samples}

Total 164 tissue samples were used for this study, including 150NSCLC paired with the adjacent non-tumor tissues and 14 normal lung tissues (from autopsy cases). All of these tissues were collected from Shanghai Outdo Biotech Co. Ltd. (Outdo Biotech) and Tissue Bank of
Yanbian University Medical College. All tissues were routinely fixed in $10 \%$ buffered formalin and embedded in paraffin blocks. The study protocol was approved by the institutional review board of Yanbian University Medical College. The pathological parameters, including gender, age, tumor size, clinical stage, differentiation, nodal metastasis and survival data, were carefully reviewed in all 150 NSCLC cases.

The patients with NSCLC including 112 males and 38 females, and ranging from 43 to 76 years with a mean age of 62 years. A total of 150 patients, 99 cases were 60 years old or over, and 51 cases were below 60 years old. All cases were confirmed with NSCLC by pathological examination. TNM staging was assessed according to the staging system established by the American Joint Committee on Cancer (AJCC). Of the 150 NSCLC, 98 cases were stages I-II while 52 cases were stages III-IV, and for the tumor sizes, 119 cases were defined as T1-T2 and 31 cases were T3-T4. In addition, 34 cases were defined as well differentiated, while 89 cases as moderately and 27 cases as poorly differentiated. Additionally, 96 cases have lymph node (LN) metastasis, and 54 cases have no LN metastasis. None of the patients received radiochemotherapy before surgery. The 150 patients with NSCLC had been followed for eight years or until death. In this study, 150 cases of adjacent non-tumor lung tissues from the cancer resection margin and 14 cases of normal lung tissues were also included.

\section{Immunofluorescence (IF) staining for NQO1 protein in A549 lung cancer cells}

Lung cancer cell line A549 was grown on coverslips to $70 \%$ confluence, then all cells were fixed with $4 \%$ paraformaldehyde for 10 minutes and permeabilized with $0.5 \%$ TritonX-100 for 10 minutes after 24 hours. Blocking was performed with 3\% Albumin Bovine V (A8020, Solarbio, Beijing, China) for 1 hour at the room temperature (RT). After washing with PBS, cells were incubated with antibody against NQO1 (1:200, Cell Signaling Technology, Boston, USA) for 2 hours at $37^{\circ} \mathrm{C}$, and followed the incubation by Alexa Fluor 488 goat anti-rabbit IgG $(\mathrm{H}+\mathrm{C})(\mathrm{A} 11008$, Invitrogen, USA) respectively, for 1 hour at RT. After washing with PBS, cells were counterstained with 49-6-diamidino-2-phenylindole (DAPI) (C1006, Beyotime, Shanghai, China) and the coverslips were mounted with Antifade Mounting Medium (P0126, Beyotime, Shanghai, China). Finally, the immunofluorescence signals were visualized and recorded by Leica SP5II confocal microscope.

\section{Immunohistochemistry (IHC) for NQO1 protein in paraffin-embedded tissues}

IHC analysis was performed using the DAKO LSAB kit (DAKO A/S, Glostrup, Denmark). Briefly, to eliminate 
endogenous peroxidase activity, $4 \mu \mathrm{m}$ thick tissue sections were deparaffinized, rehydrated and incubated with $3 \% \mathrm{H}_{2} \mathrm{O}_{2}$ in methanol for $15 \mathrm{~min}$ at RT. The antigen was retrieved at $95^{\circ} \mathrm{C}$ for $20 \mathrm{~min}$ by placing the slides in $0.01 \mathrm{M}$ sodium citrate buffer ( $\mathrm{pH}$ 6.0). The slides were then incubated with NQO1 antibody (1:600, BD Biosciences Pharmingen, CA, USA) at $4^{\circ} \mathrm{C}$ overnight. After incubation with biotinylated secondary antibody at RT for $30 \mathrm{~min}$, the slides were incubated with streptavidinperoxidase complex at RT for $30 \mathrm{~min}$. IHC staining was developed by using 3,3'-diaminobenzidine, and Mayer's hematoxylin was used for counterstaining. In addition, the positive tissue sections were processed with omitting of the primary antibody as negative controls.

\section{Evaluation of IHC staining}

All specimens were examined by two investigators (Jin T \& Lin Z) who did not possess knowledge of the clinical data. In case of discrepancies, a final score was established by reassessment on a double-headed microscope. Briefly, the IHC staining for NQO1 was semi-quantitatively scored as '-' (negative, no or less than 5\% positive cells), ' + ' (5$50 \%$ positive cells), and ' ++ ' (more than $50 \%$ positive cells, considered as strongly positive). Only the cytoplasmic expression pattern was considered as positive staining.

\section{Statistical analysis}

Statistical analyses were performed using the SPSS software program for windows, version 17.0 (SPSS, Inc., Chicago, IL, USA). Correlation between NQO1 expression and clinicopathological characteristics were evaluated by Chi-square test and Fisher's exact tests. The survival rates after tumor removal were calculated by the Kaplan-Meier method, and differences in survival curves were analyzed by the Log-rank tests. Multivariate survival analysis was performed on all the significant characteristics measured by univariate survival analysis through the Cox proportional hazard regression model. P-values less than 0.05 were considered statistically significant.

\section{Results}

High expression of NQO1 protein in NSCLC

IF staining indicated that NQO1 protein was mainly located in the cytoplasm of A549 lung cancer cells (Figure 1). IHC staining consistently showed that the NQO1 protein was located in the cytoplasm of lung SCC and adenocarcinoma (Figure 2B \& D). The positive rate of the NQO1 protein expression was $59.3 \%(89 / 150)$ in NSCLC tissues, which was significantly higher than that in adjacent non-tumor $(8.0 \%, 12 / 150)$, and the expression were all negative in normal lung tissues $(P<0.01)$. Similarly,
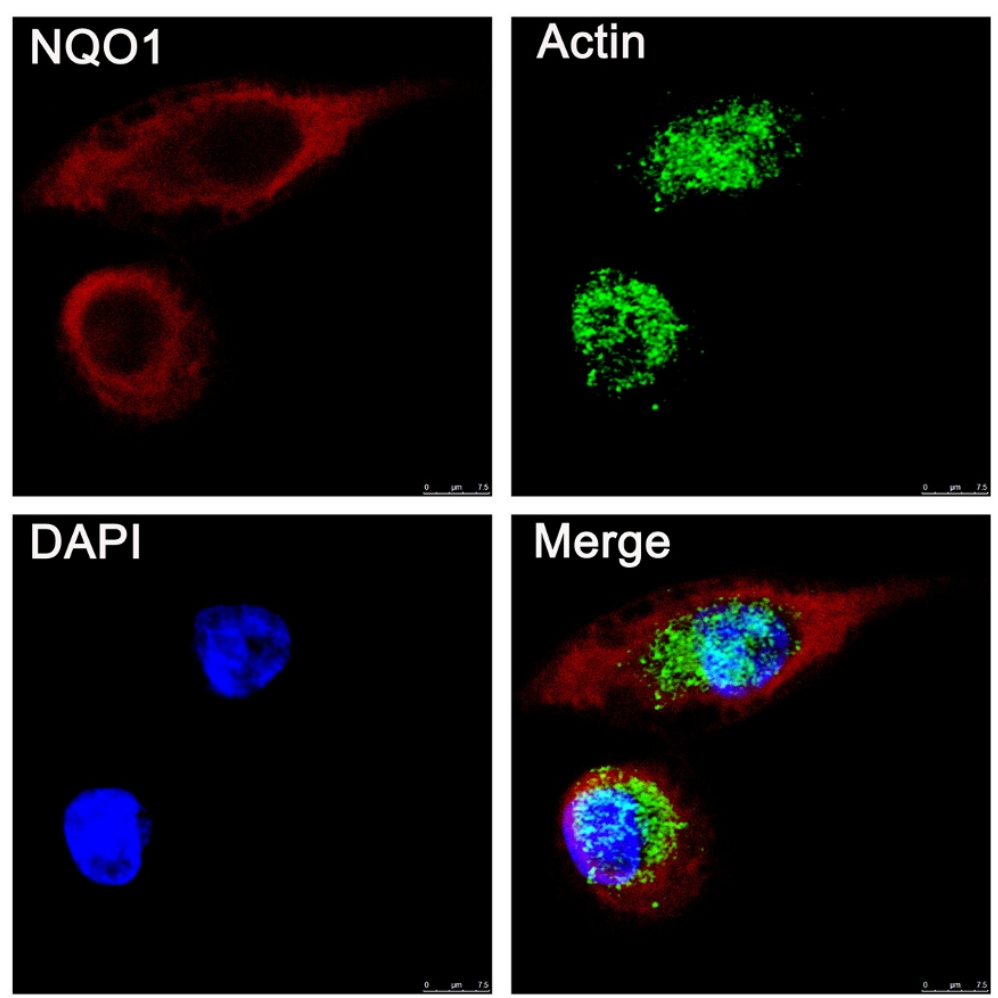

Figure $1 \mathrm{IF}$ staining for NQO1 protein in A549 human lung cancer cells. NQO1 protein located in the cytoplasm of A549 cells (Red for NQO1, Green for Actin, and Blue for DAPI). 


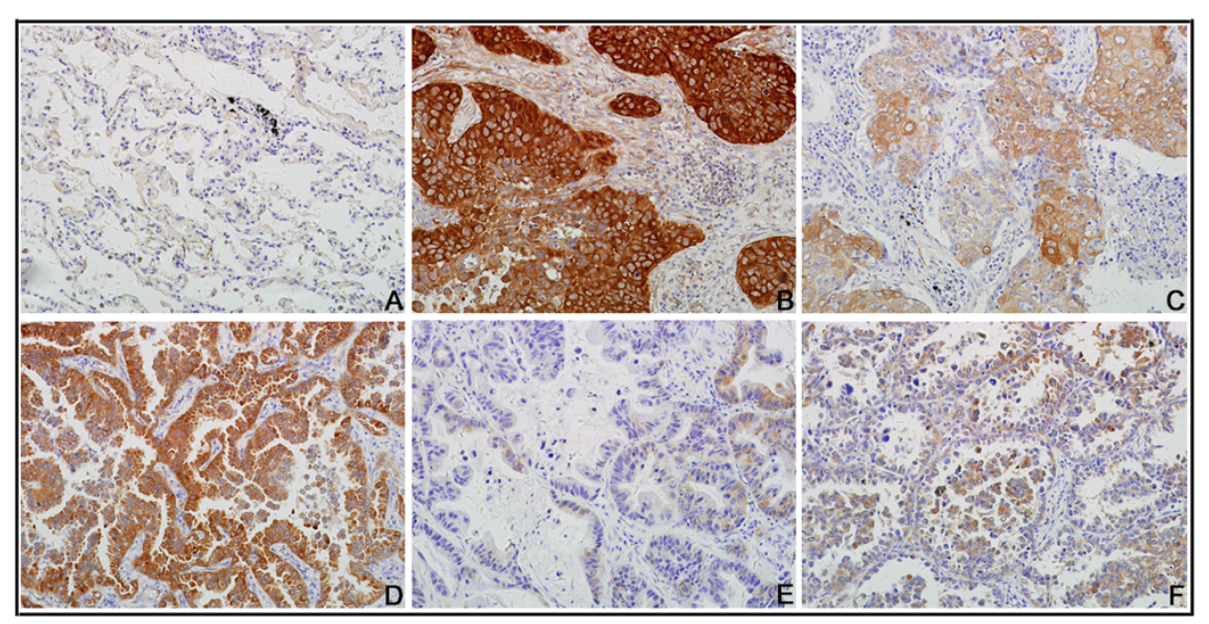

Figure 2 IHC staining for NQO1 protein expression in lung tissues. (A) NQO1 protein was negative in normal lung tissues. (B) NQO1 protein was showed diffuse and strong positive staining in cytopalsm of lung SCC cells with LN metastasis. (C) NQO1 was weakly positive in lung SCC without LN metastasis. (D) Diffuse and strong positive NQO1 protein signal in lung adenocarcinoma. (E \& F) NQO1 protein staining is negative or weakly positive in lung adenocarcinoma. (Original magnification, 200x in A-F).

the strongly positive rate of NQO1 expression was $28.0 \%$ $(42 / 150)$ in NSCLC, which was also significantly higher than that in adjacent non-tumor $(1.3 \%, 2 / 150)$ $(P<0.01)$ (Table 1).

\section{Clinicopathological significance of NQO1 expression in patients with NSCLC}

The relationship between NQO1 protein and the clinicopathological parameter of NSCLC was analyzed. The positive rate of NQO1 protein was related with clinical stage and lymph node metastasis. Moreover, the strongly positiverate of NQO1 protein was significantly higher in NSCLC with T3-4 $(>5 \mathrm{~cm})$ tumor size than in cases with T1-2 $(\leq 5 \mathrm{~cm})$ tumor size $(P=0.005)$. Similarly, we found that the strongly positive rate of NQO1 protein was significantly higher in stages III-IV $(36.54 \%, 19 / 52)$ than those in stages I-II $(23.47 \%, 23 / 98)(P=0.003)$. Also, it was higher in poorly differentiated NSCLC $(55.56 \%, 15 /$ $27)$ than in moderately $(31.46 \%, 28 / 89)$ and well differentiated NSCLC $(26.47 \%, 9 / 34)(P=0.012)$. Additionally, it was also higher in NSCLC patients with lymph node metastasis $(50.00 \%, 27 / 54)$ than in cases without metastasis $(15.63 \%, 15 / 96)(P=0.000)$. However, there was no significant correlations between high-level NQO1 expression and gender, and age of patients with NSCLC $(P>0.05$, respectively) (Table 2$)$.

To further substantiate the importance of NQO1 expression in NSCLC progression, we analyzed the relationships between NQO1 positive expression rate and DFS and OS in 150 lung cancer cases using the KaplanMeier method, and found that patients with NQO1 positive expression had lower DFS (Log-rank $=13.899, P<$ 0.001 ) and $\mathrm{OS}$ (Log-rank $=10.146, P=0.001)$ rates than those with NQO1 negative expression (Figure 3A \& B).

Similarly, we also analyzed the association between the NQO1 expression and tumor size, lymph node metastasis, and clinical stages of NSCLC. The patients with NQO1 positive expression had lower OS rates compared with those with NQO1 negative expression in the groups of T1-2 (Log-rank $=9.931, P=0.002$ ), T3-4 (Log-rank=9.387, $P=0.002)$ (Figure 4A \& B), without LN metastasis (Log-rank $=9.274, \quad P=0.002$ ) and stage I-II of NSCLC (Log-rank $=5.770, P=0.016$ ) (Figure $4 \mathrm{C} \& \mathrm{E}$ ), however, in the groups of patients with LN metastasis or III-IV stages, OS rate was not correlated with NQO1 expression status (Log-rank = $0.919, P=0.553$ and Log-rank $=0.572, P=0.050$, respectively) (Figure 4D \& F).

Table 1 NQO1 protein expression in NSCLC

\begin{tabular}{|c|c|c|c|c|c|c|}
\hline \multirow[t]{2}{*}{ Diagnosis } & \multirow{2}{*}{$\begin{array}{l}\text { No. of } \\
\text { cases }\end{array}$} & \multicolumn{3}{|c|}{ NQ01 protein expression } & \multirow{2}{*}{$\begin{array}{l}\text { Positive rate } \\
(+\sim++)\end{array}$} & \multirow{2}{*}{$\begin{array}{l}\text { Strongly positive } \\
\text { rate }(++)\end{array}$} \\
\hline & & - & + & ++ & & \\
\hline$\overline{\mathrm{NSCLC}}$ & 150 & 61 & 47 & 42 & $59.3 \% * *$ & $28.0 \% * *$ \\
\hline Adjacent non tumor & 150 & 138 & 10 & 2 & $8.0 \%$ & $1.3 \%$ \\
\hline Normal lung tissues & 14 & 14 & 0 & 0 & 0 & 0 \\
\hline
\end{tabular}

${ }^{*} P<0.01$ compared with normal lung tissues and adjacent non tumor tissues. 
Table 2 Correlation between NQO1 expression and clinicopathological features of NSCLC

\begin{tabular}{|c|c|c|c|c|c|}
\hline \multirow[t]{2}{*}{ Variables } & \multirow[t]{2}{*}{ Case no. } & \multicolumn{2}{|c|}{ NQ01 positive $(+\sim++)$} & \multicolumn{2}{|c|}{ NQ01 strongly positive(++) } \\
\hline & & n (\%) & $P$ value & n (\%) & $P$ value \\
\hline Gender & & & 0.579 & & 0.570 \\
\hline Male & 112 & $65(58.04)$ & & $30(26.79)$ & \\
\hline Female & 38 & $24(63.16)$ & & 12(31.58) & \\
\hline Age & & & 0.541 & & 0.914 \\
\hline$\geqq 60$ & 99 & $57(57.58)$ & & $28(28.28)$ & \\
\hline$<60$ & 51 & $32(62.75)$ & & $14(27.45)$ & \\
\hline Tumor size & & & 0.567 & & $0.005^{* *}$ \\
\hline $\mathrm{T} 1-2$ & 119 & $72(60.50)$ & & $27(22.69)$ & \\
\hline T3-4 & 31 & $17(54.84)$ & & 15(48.39) & \\
\hline Stage & & & $0.013^{*}$ & & $0.003^{* *}$ \\
\hline$|-| \mid$ & 98 & $51(52.04)$ & & $23(23.47)$ & \\
\hline III-IV & 52 & $38(73.08)$ & & 19(36.54) & \\
\hline Differentiation & & & 0.085 & & $0.012^{*}$ \\
\hline Well & 34 & $15(44.12)$ & & $9(26.47)$ & \\
\hline Moderately & 89 & $54(60.67)$ & & 28(31.46) & \\
\hline Poorly & 27 & $20(74.07)$ & & 15(55.56) & \\
\hline LN metastasis & & & $0.039^{*}$ & & $0.000^{* *}$ \\
\hline \multirow[t]{2}{*}{ Negative Positive } & 96 & $51(53.13)$ & & 15(15.63) & \\
\hline & 54 & $38(70.37)$ & & $27(50.00)$ & \\
\hline
\end{tabular}

${ }^{*} P<0.05,{ }^{* *} P<0.01$.

NQ01 expression is an independent prognostic biomarkerin NSCLC by Cox proportional hazardsregression model

Univariate analysis demonstrated that the NSCLC patients with NQO1 positive expression had significant lower OS rate (HR: 1.442 , 95\% CI: $1.036-2.007, P=0.030$ ) than those with NQO1 negative expression. Additionally, age (HR: 1.498, 95\% CI: 1.062-2.113, $P=0.021$ ), tumor size (HR: 5.566, 95\% CI: 3.499-8.857, $P=0.000$ ), differentiation (HR: 1.426, 95\% CI: 1.101-1.847, $P=0.007$ ), lymph node metastasis (HR: 2.300, 95\% CI: 1.607-3.292, $P=$ 0.000)and clinical stage (HR: 3.720, 95\% CI: 2.526-5.477, $P=0.000)$ were all significantly associated with OS rates of NSCLC patients. Then, multivariate analysis was performed using the Cox proportional hazards model for all of the significant variables, which were examined in the univariate survival analysis. We found that NQO1 expression emerged as a significant independent prognostic factor for OS rates in patients with NSCLCs (HR: 1.514, 95\% CI: $1.066-2.151, P=0.020)$ along with tumor
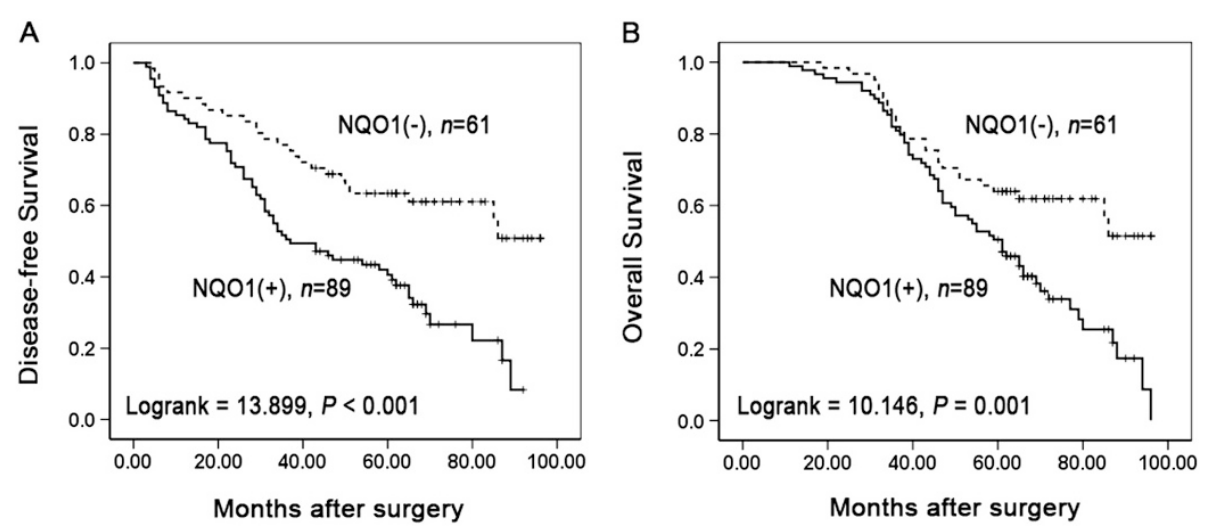

Figure 3 Kaplan-Meier analysis of DFS and OS rates in 150 NSCLC patients in relation to NQO1 protein expression. Patients of NSCLC with NQO1 positive expression had lower DFS $(\mathbf{A}, P<0.001)$ and OS $(\mathbf{B}, P<0.001)$ rates than those with NQO1 negative expression $(+$, positive expression; -, negative expression). 

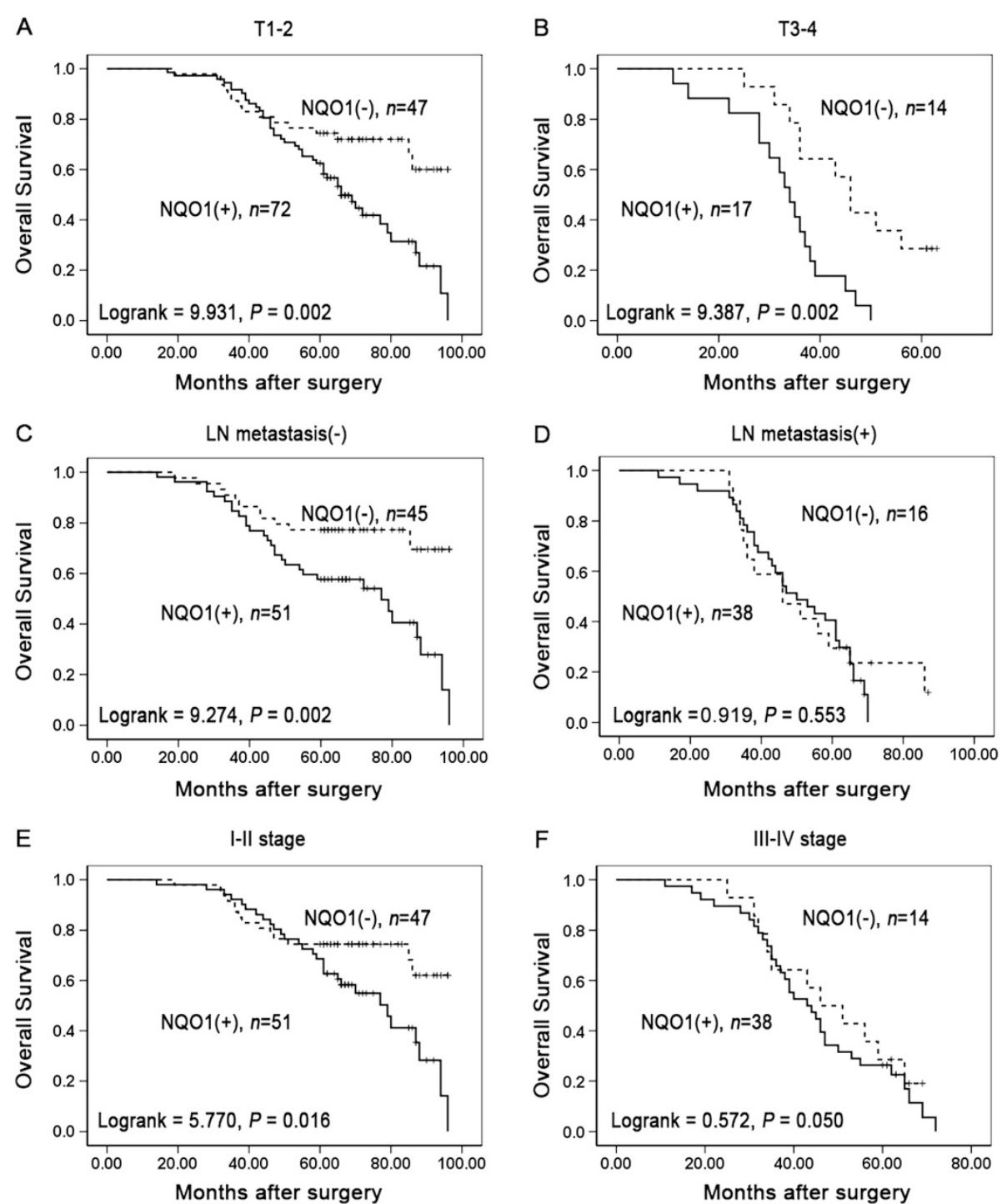

Figure 4 Kaplan-Meier analysis of OS rates in patients with or without NQO1 expressed NSCLC in prognostic factors. OS was assessed in NSCLC patients with T1-2 (A, $P=0.002)$, T3-4 (B, $P=0.002)$, LN metastasis $(-)(\mathbf{C}, P=0.002)$, LN metastasis $(+)(\mathbf{D}, P=0.553), I-I I$ stage $(\mathbf{E}, P=0.016)$, and III-IV stage $(\mathbf{F}, P=0.050)$ concomitant with either positive- or negative-expression of NQO1.

size (HR: 5.545, 95\% CI: 3.283-9.366, P =0.000), differentiation (HR: 1.369, 95\% CI: 1.055-1.775, $P=0.018$ ), lymph node metastasis (HR: 1.962, 95\% CI: 1.334-2.884, $P=0.001)$ and clinical stage (HR: 2.192 , 95\% CI: $1.403-$ 3.425, $P=0.001$ ) (Table 3).

\section{Discussion}

NQO1, known as NAD(P)H: quinone oxidoreductase-1, was first identified by Ernster and Navazio in 1958 [14]. NQO1 is a homodimericflavoprotein and many functions have been proposed, such as xenobiotic detoxification, superoxide scavenging, modulation of p53, maintenance of endogenous antioxidants, and proteasomal degradation [6]. Several studies have indicated that the phase II enzyme NQO1 catalyzes the metabolic detoxification of quinones and protects cells against chemical-induced oxidative stress and cancer $[15,16]$. Nagata et al. [17] and Malik et al. [18] reported that the C609T polymorphism in the NQO1 gene affects the translation of the NQO1 protein, and have been reported to be associated with an increased risk of cancers death. Moreover, NQO1 polymorphism that leads to the enzyme inactivity has been found to be a strong prognostic and predictive factor in the poor outcome of breast cancer [19]. NQO1 has also been shown to act as a chaperone, thereby stabilizing various proteins, including the tumor suppressor protein p53 [20] and other short-lived proteins such as ornithine decarboxylase [21]. These studies suggested that NQO1 activities may be essential for cancer progression. 
Table 3 Univariateand multivariate analysis of clinicopathological factors for the overall survival rate of 150 patients with NSCLC

\begin{tabular}{lllll}
\hline Characteristics & Univariate analysis HR $(\mathbf{9 5} \% \mathrm{Cl})$ & $\boldsymbol{P}$ value & Multivariate analysis HR $\mathbf{( 9 5 \% \mathrm { Cl } )}$ & $\boldsymbol{P}$ value \\
\hline Gender & $1.047(0.724-1.513)$ & 0.809 & $1.023(0.700-1.496)$ & 0.907 \\
Age & $1.498(1.062-2.113)$ & $0.021^{*}$ & $1.050(0.728-1.516)$ & 0.793 \\
Tumor size & $5.566(3.499-8.857)$ & $0.000^{* *}$ & $5.545(3.283-9.366)$ & $0.000^{* *}$ \\
Differentiation & $1.426(1.101-1.847)$ & $0.007^{* *}$ & $1.369(1.055-1.775)$ & $0.018^{*}$ \\
LN metastasis & $2.300(1.607-3.292)$ & $0.000^{* *}$ & $1.962(1.334-2.884)$ & $0.001^{* *}$ \\
Stage & $3.720(2.526-5.477)$ & $0.000^{* *}$ & $2.192(1.403-3.425)$ & $0.001^{* *}$ \\
NQO1 & $1.442(1.036-2.007)$ & $0.030^{*}$ & $1.514(1.066-2.151)$ & $0.020^{*}$ \\
\hline
\end{tabular}

LN: lymph node; HR: hazard ratio; Cl: confidence interval.

${ }^{*} P<0.05$, ${ }^{* *} P<0.01$.

Accumulating studies showed that NQO1 was expressed at relatively high levels in many solid tumors. For example, our previous study [22] demonstrated that NQO1 protein expression was significantly elevated in breast cancer tissues compared with hyperplasia or adjacent non-tumor tissues, indicating that NQO1 upregulation may occur in the initiation stage of breast cancer progression. Similarly, compared with normal cervical epithelia, the strongly positive rate of NQO1 protein expression was also significantly higher in cervical SCC and intraepithelial neoplasia tissues, indicating that NQO1 expression might be related to tumorigenesis of cervical cancer [23]. Furthermore, we also found that NQO1 protein was frequently high-expressed in gastric adenocarcinoma compared with the gastric dysplasia and adjacent non-tumor tissues, indicating that NQO1 was a significant prognostic or predictive maker of gastric adenocarcinoma [24]. Consistently, Awadallah et al. [25] and Lyn-Cook et al. [26] reported that NQO1 protein was up-regulated in pancreatic ductal adenocasinoma, and also considered that NQO1 may represent a role of useful biomarker for pancreatic cancer. Malkinson et al. [27] found that NQO1 gene was observed to be high-expressed in human lung cancer tissues, and Rosvold et al. [28] and Heller et al. [29] also indicated that the gene encoding NQO1 is a promising candidate in the pathogenesis of lung cancer. However, to date, the clinicopathological significance of NQO1 protein expression in NSCLC has not been elucidated.

Thus, here we performed IF and IHC staining in 150 NSCLC paired with the adjacent non-tumor tissues and 14 normal lung tissues, and found that NQO1 protein localized in the cytoplasm of A549 lung cancer cells and NSCLC tissues. Both positive and strongly positive rates of NQO1 protein expression were significantly higher than both in adjacent non-tumor and normal lung tissues. These results indicate that NQO1 played an important role in the progression of lung cancer. Mikami $\mathrm{K}$ et al. [30] reported that the expression and enzyme activity of NQO1 was up-regulated in colon cancer cell lines and colorectal tumors, and moreover significantly higher in tumors with LN metastases than those without metastasis. Here we analyzed the correlation between NQO1 expression and clinicopathological parameters of NSCLC, and the results showed that NQO1 expression and highexpression was all significantly associated with LN metastasis and clinical stage. Moreover, the strongly positive rate of NQO1 protein was higher in NSCLCs with larger tumor size $(>5 \mathrm{~cm})$ than in cases with smaller $(\leq 5 \mathrm{~cm})$, and it was also significantly higher in poorly differentiated NSCLC than in moderately and well differentiated NSCLC. These results indicated that NQO1 might be a predictive biomarker for poor prognostic evaluation of NSCLCs, and NQO1 protein maybe participated in the tumorigenesis and malignant progression of NSCLC.

In regard to survival, we previously found that high expression of NQO1 protein was strongly associated with advanced stage, lymph node metastasis, Her2 overexpression and shortened survival of patients with breast cancer [22]. Moreover, Buranrat et al. [12] also reported a significant association between high level of NQO1 expression and short overall survival time of cholangiocarcinoma patients, which raised the exciting possibility of using NQO1 as a tumor marker. However, Kim et al. [31] reported that there was no correlation between NQO1 and prognosis of small-cell lung cancer. Here we found that NSCLC patients with NQO1 protein positiveexpression had a lower DFS and OS rates than those with NQO1 protein negative-expression. Additionally, age, tumor size, differentiation, lymph node metastasis, clinical stage and NQO1 expression were all significantly associated with OS rates of NSCLC patients $(P<0.05)$. Furthermore, multivariate survival analysis demonstrated that NQO1 positive-expression was an independent prognostic factor along with tumor size, differentiation, lymph node metastasis and clinical stage. These findings indicated that NQO1 might be a potentially predictive biomarker of poor prognosis, especially in patients with poor differentiation, lymph node metastasis and clinical stage of NSCLC. 
Recently, NQO1 has been used as the target enzyme in tumor cells to exemplify the 'enzyme directed' approach to anticancer drug development [32]. Park et al. [33] and Kung et al. [34] demonstrated that NQO1 bioactivatable drugs ( $\beta$-Lapachone or deoxynyboquinone [DNQ]) can effectively kill the cancer cells. Huang et al. [35] reported that the potency and NQO1-dependent therapeutic window of DNQ and its apparent reduced metabolism by one-electron oxidoreductases make this drug (or derivatives) very promising. Therefore, the further study will be significant to verify if the NQO1 inhibitor could be used for the therapy of patients with NSCLC.

\section{Conclusions}

In conclusion, NQO1 is frequently upregulated in NSCLC, and it may be a useful poor prognostic biomarker and a potential therapeutic target for patients with NSCLC.

\section{Abbreviations}

NQO1: NAD(P)H quinone oxidoreductase 1; NSCLC: Non-small cell lung cancer; SCC: Squamous cell carcinoma; EGFR: Epidermal growth factor receptor; EML4-ALK: Echinoderm microtubule associated protein like4-anaplastic lymphoma kinase; DNQ: Deoxynyboquinone.

\section{Competing interests}

The authors declare that they have no competing interests.

\section{Authors' contributions}

$Z L, Y Z, T J$ and YP participated in the study conception, design, case selection and experiments. ZL, JM and PQ carried out data collection. GY, SP and ZL performed the data analysis and wrote the manuscript. All authors read and approved the final manuscript.

\section{Acknowledgments}

This study was supported by grants from National Natural Science Funds of China (81260665), and The Projects of Research \& Innovation of Jilin Youth Leader and Team, China (20140519013JH).

\section{Author details}

1Department of Pathology \& Cancer Research Center, Yanbian University Medical College, Yanji 133002, China. ${ }^{2}$ Department of TCM, Jilin Cancer Hospital, Changchun 130012, China. ${ }^{3}$ Department of Anatomy and Histology and Embryology, Yanbian University Medical College, Yanji 133002, China. ${ }^{4}$ Department of Pathophysiology, Yanbian University Medical College, Yanji 133002, China.

Received: 7 September 2014 Accepted: 19 March 2015

Published online: 31 March 2015

\section{References}

1. Siegel R, Naishadham D, Jemal A. Cancer statistics, 2013. CA Cancer J Clin. 2013;63(1):11-30

2. Chen X, Liu Y, Røe OD, Oian Y, Guo R, Zhu L, et al. Gefitinib or erlotinib as maintenance therapy in patients with advanced stage non-small cell lung cancer: a systematic review. PLoS One. 2013;8(3):e59314.

3. Gaughan EM, Costa DB. Genotype-driven therapies for non-small cell lung cancer: focus on EGFR, KRAS and ALK gene abnormalities. Ther Adv Med Oncol. 2011;3(3):113-25.

4. Jin T, Jin J, Shang Y, Zhang S, Yu K, Piao Y, et al. Prognostic implications of ezrin and phosphorylated ezrin expression in non-small cell lung cancer. BMC Cancer. 2014;14:191.

5. Vasilious $\mathrm{V}$, Rose $\mathrm{D}$, Nebert DW. Update of the $\mathrm{NAD}(\mathrm{P}) \mathrm{H}$ : quinone oxidoreductase (NQO) gene family. Hum Genomic. 2006;2(5):329-35.

6. Buranrat B, Prawan A, Kukongviriyapan U, Kongpetch S, Kukongviriyapan V. Dicoumarol enhances gemcitabine-induced cytotoxicity in high
NQO1-expressing cholangiocarcinoma cells. World J Gastroenterol. 2010;16(19):2362-70

7. Siegel D, Gustafson DL, Dehn DL, Han JY, Boonchoong P, Berliner $L$, et al. $\mathrm{NAD}(\mathrm{P}) \mathrm{H}$ :quinone oxidoreductase 1: role as a superoxide scavengar. Mol Pharmacol. 2004;65(5):1238-47.

8. Siegel D, Bolton EM, Burr JA, Liebler DC, Ross D. The reduction of alpha-tocopherolquinone by human $\mathrm{NAD}(\mathrm{P}) \mathrm{H}$ : quinone oxidoreductase: the role of alpha-tocopherolhydrolquinone as a cellular antioxidant. Mol Pharmacol. 1997;52(2):300-5.

9. Siegel D, Ross D. Immunodetection of $\mathrm{NAD}(\mathrm{P}) \mathrm{H}$ : quinone oxidoreductase 1 (NQO1) in human tissues. Free RadicBiol Med. 2000;29(2-3):246-53.

10. Chao C, Zhang ZF, Berthiller J, Boffetta P, Hashibe M. NAD(P)H:quinone oxidoreductase 1 (NQO1) Pro1875er polymorphism and the risk of lung, bladder, and colorectal cancer: a meta-analysis. Cancer Epidemiol Biomarkers Prev. 2006;15(5):979-87.

11. Awadallah NS, Dehn D, Shah RJ, Russell Nash S, Chen YK, Ross D, et al. NQO1 expression in pancreatic cancer and its potentail use as a biomarker Appllmmunohistochem Mol Morphol. 2008;16(1):24-31.

12. Buranrat B, Chau-in S, Prawan A, Puapaoroj A, Zeekpudsa P, Kukongviriyapan V. NQ01 expression correlates with cholangiocarcinoma prognosis. Asian Pac J Cancer Pre. 2012:13(Suppl):131-6.

13. Garate $M$, Wani AA, Li G. The NAD(P)H:Quinone Oxidoreductase 1 inducescell cycle progression and proliferation of melanoma cells. Free RadicBiolMed. 2010;48(12):1601-9.

14. Ernster $L$, Lindberg O. Animal mitochondria. Annu Rev Physiol. 1958;20:13-42

15. Nioi $\mathrm{P}$, Hayes JD. Contribution of $\mathrm{NAD}(\mathrm{P}) \mathrm{H}$ : quinone oxidoreductase 1 to protection against carcinogenesis, and regulation of its gene by the Nrf2 basic-region leucine zipper and the arylhydrocarbon receptor basic helix-loop-helix transcription factors. Mutat Res. 2004;555(1-2):149-71.

16. Girolami F, Abbadessa G, Racca S, Spaccamiglio A, Piccione F, Dacasto M, et al. Time-dependent acetylsalicylic acid effects on liver CYP1A and antioxidant enzymes in a rat model of 7,12-dimethylbenzanthracene (DMBA)-induced mammary carcinogenesis. Toxicol Lett. 2008;181(2):87-92

17. Nagata M, Kimura T, Suzumura T, Kira Y, Nakai T, Umekawa K, et al. C609Tpolymorphism of NADPH Quinone Oxidoreductase 1 Correlates clinicalhematological toxicities in lung cancer patients treated with Amrubicin. Clin Med Insights Oncol. 2013;7:31-9.

18. Malik MA, Zargar SA, Mittal B. Role of NQO1 609C > T and NQO2-3423G > A gene polymorphisms in esophageal cancer risk in Kashmir valley andmeta analysis. Mol Biol Rep. 2012;39(9):9095-104

19. Fagerholm R, Hofstetter B, Tommiska J, Aaltonen K, Vrtel R, Syrjäkoski K, et al. $\mathrm{NAD}(\mathrm{P}) \mathrm{H}$ : quinone oxidoreductase $1 \mathrm{NQO}^{*} 2$ genotype (P187S) is a strong prognostic and predictive factor in breast cancer. Nat Genet. 2008:40(7):844-53.

20. Asher G, Lotem J, Cohen B, Sachs L, Shaul Y. Regulation of p53 stability and p53-dependent apoptosis by NADH quinoneoxidoreductase 1. Proc NatlAcadSci U S A. 2001;98(3):1188-93.

21. Asher G, Bercovich Z, Tsvetkov P, Shaul Y, Kahana C. 20 S proteasomal degradation of ornithine decarboxylase is regulated by NQO1. Mol Cell. 2005;17(5):645-55

22. Yang Y, Zhang Y, Wu Q, Cui X, Lin Z, Liu S, et al. Clinical implications of high NQ01 expression inbreast cancers. J Exp Clin Cancer Res. 2014;33:14

23. Ma Y, Kong J, Yan G, Ren X, Jin D, Jin T, et al. NQO1 overexpression is associated with poor prognosis in squamous cell carcinoma of the uterine cervix. BMC Cancer. 2014;14:414.

24. Lin L, Qin Y, Jin T, Liu S, Zhang S, Shen X, et al. Significance of NQO1overexpression for prognostic evaluation of gastric adenocarcinoma. Exp Mol Pathol. 2014;96(2):200-5.

25. Awadallah NS, Dehn D, Shah RJ, Russell Nash S, Chen YK, Ross D, et al. NQO1 expression in pancreatic cancer and its potential use as a biomarker Appl Immunohistochem Mol Morphol. 2008;16(1):24-31.

26. Lyn-Cook BD, Yan-Sanders Y, Moore S, Taylor S, Word B, Hammons GJ. Increased levels of $\mathrm{NAD}(\mathrm{P}) \mathrm{H}$ : quinoneoxidoreductase 1 (NQO1) in pancreatic tissues from smokers and pancreatic adenocarcinomas: A potential biomarker of early damage in the pancreas. Cell Biol Toxicol. 2006;22(2):73-80

27. Malkinson AM, Siegel D, Forrest GL, Gazdar AF, Oie HK, Chan DC, et al. Elevated DT-diaphorase activity and messenger RNA content in human non-small cell lung carcinoma: relationship to the response of lung tumor xenografts to mitomycin Cl. Cancer Res. 1992;52(17):4752-7. 
28. Rosvold EA, McGlynn KA, Lustbader ED, Buetow KH. Identification of an $\mathrm{NAD}(\mathrm{P}) \mathrm{H}$ : quinone oxidoreductase polymorphism and its association with lung cancer and smoking. Pharmacogenetics. 1995;5(4):199-206.

29. Heller G, Zielinski CC, Zochbauer-Muller S. Lung cancer: from single-gene methylation to methylome profiling. Cancer Metastasis Rev. 2010;29(1):95-107.

30. Mikami $K$, Naito M, Ishiguro $T$, Yano $H$, Tomida $A$, Yamada $T$, et al. Immunological quantitation of DT-diaphorase in carcinoma cell lines and clinical colon cancers: advanced tumors express greater levels of DT-diaphorase. Jpn J Cancer Res. 1998;89(9):910-5.

31. Kim HC, Song JS, Lee JC, Lee DH, Kim SW, Lee JS, et al. Clinical significance of NQO1 polymorphism and expression of p53, SOD2, PARP1 in limitedstage small cell lung cancer. Int J Clin Exp Pathol. 2014;7(10):6743-51.

32. Workman P. Enzyme-directed bioreductive drug development revisited: a commentary on recent progress and future prospects with emphasis on quinone anticancer agents and quinone metabolizing enzymes, particularly DT-diaphorase. Oncol Res. 1994;6(10-11):461-75.

33. Park EJ, Min KJ, Lee TJ, Yoo YH, Kim YS, Kwon TK. $\beta$-Lapachoneindcuces programmed necrosis through the RIP1-PARP-AIF-dependent pathway in human hepatocellular carcinoma SK-Hep1 cells. Cell Death Dis. 2014;5:e1230.

34. Kung H, Weng T, Liu Y, Lu K, Chau Y. Sulindac Compounds Facilitate the Cytotoxicity of $\beta$-Lapachone by Up-Regulation of NAD(P)H Quinone Oxidoreductase in Human Lung Cancer Cells. PLoS One. 2014;9(2):e88122.

35. Huang X, Dong Y, Bey E. An NQO1 Substrate with Potent Antitumor Activity That Selectively Kills by PARP1-Induced Programmed Necrosis. Cancer Res. 2012;72(12):3038-47.

\section{Submit your next manuscript to BioMed Central and take full advantage of:}

- Convenient online submission

- Thorough peer review

- No space constraints or color figure charges

- Immediate publication on acceptance

- Inclusion in PubMed, CAS, Scopus and Google Scholar

- Research which is freely available for redistribution 